\title{
The Riemann Hypothesis Is True
}

\author{
Frank Vega
}

the date of receipt and acceptance should be inserted later

\begin{abstract}
The Riemann hypothesis is a conjecture that the Riemann zeta function has its zeros only at the negative even integers and complex numbers with real part $\frac{1}{2}$. The Riemann hypothesis belongs to the David Hilbert's list of 23 unsolved problems. Besides, it is one of the Clay Mathematics Institute's Millennium Prize Problems. This problem has remained unsolved for many years. The Robin criterion states that the Riemann hypothesis is true if and only if the inequality $\sigma(n)<e^{\gamma} \times n \times \log \log n$ holds for all natural numbers $n>5040$, where $\sigma(x)$ is the sum-of-divisors function and $\gamma \approx 0.57721$ is the Euler-Mascheroni constant. The Nicolas criterion states that the Riemann hypothesis is true if and only if the inequality $\prod_{q \leq q_{n}} \frac{q}{q-1}>e^{\gamma} \times \log \theta\left(q_{n}\right)$ is satisfied for all primes $q_{n}>2$, where $\theta(x)$ is the Chebyshev function. Using both inequalities, we show that the Riemann hypothesis is true.
\end{abstract}

Keywords Robin inequality · Nicolas inequality · sum-of-divisors function · Chebyshev function · prime numbers $\cdot$ Riemann zeta function

Mathematics Subject Classification (2010) MSC 11M26 · MSC 11A41 · MSC $11 \mathrm{~A} 25$

\section{Introduction}

In mathematics, the Chebyshev function $\theta(x)$ is given by

$$
\theta(x)=\sum_{q \leq x} \log q
$$

where $q \leq x$ means all the prime numbers $q$ that are less than or equal to $x$. Let $N_{n}=2 \times 3 \times 5 \times 7 \times 11 \times \cdots \times q_{n}$ denotes a primorial number of order $n$ such that $q_{n}$

F. Vega

CopSonic, 1471 Route de Saint-Nauphary 82000 Montauban, France

ORCiD: 0000-0001-8210-4126

E-mail: vega.frank@gmail.com 
is the $n^{t h}$ prime number. Thus, $\theta\left(q_{n}\right)=\log N_{n}$. We define a sequence based on this function:

Definition 1.1 For every prime number $q_{n}$, we define the sequence of real numbers:

$$
X_{n}=\frac{\prod_{q \leq q_{n}} \frac{q+1}{q}}{\log \theta\left(q_{n}\right)} .
$$

We use this limit superior,

Theorem 1.2 [1].

$$
\limsup _{n \rightarrow \infty} X_{n}=\frac{e^{\gamma} \times 6}{\pi^{2}} .
$$

Say $\operatorname{Nicolas}\left(q_{n}\right)$ holds provided

$$
\prod_{q \leq q_{n}} \frac{q}{q-1}>e^{\gamma} \times \log \theta\left(q_{n}\right) .
$$

The constant $\gamma \approx 0.57721$ is the Euler-Mascheroni constant and log is the natural logarithm. The importance of this inequality is:

Theorem 1.3 Nicolas $\left(q_{n}\right)$ holds for all prime numbers $q_{n}>2$ if and only if the Riemann hypothesis is true [4].

Besides, we define the following properties of the Riemann zeta function,

Theorem 1.4 [2].

$$
\prod_{k=1}^{\infty} \frac{q_{k}^{2}}{q_{k}^{2}-1}=\zeta(2)=\frac{\pi^{2}}{6} .
$$

Theorem 1.5 [2]. For $a \geq 1$ :

$$
\prod_{q}\left(1-\frac{1}{q^{a+1}}\right)=\frac{1}{\zeta(a+1)} .
$$

As usual $\sigma(n)$ is the sum-of-divisors function of $n[1]$ :

$$
\sum_{d \mid n} d
$$

where $d \mid n$ means the integer $d$ divides $n$ and $d \nmid n$ signifies that the integer $d$ does not divide $n$. Define $f(n)$ to be $\frac{\sigma(n)}{n}$. We know these properties for this function:

Theorem 1.6 [3]. Let $\prod_{i=1}^{m} q_{i}^{a_{i}}$ be the representation of $n$ as a product of primes $q_{1}<\cdots<q_{m}$ with natural numbers as exponents $a_{1}, \ldots, a_{m}$. Then,

$$
f(n)=\left(\prod_{i=1}^{m} \frac{q_{i}}{q_{i}-1}\right) \times \prod_{i=1}^{m}\left(1-\frac{1}{q_{i}^{a_{i}+1}}\right) .
$$


Theorem 1.7 [1]. For $n>1$ :

$$
f(n)<\prod_{q \mid n} \frac{q}{q-1}
$$

Say Robins $(n)$ holds provided

$$
f(n)<e^{\gamma} \times \log \log n .
$$

The importance of this inequality is:

Theorem 1.8 Robins $(n)$ holds for all natural numbers $n>5040$ if and only if the Riemann hypothesis is true [5]. If the Riemann hypothesis is false, then there are infinitely many natural numbers $n>5040$ such that Robins $(n)$ does not hold [5].

It is known that Robins $(n)$ holds for many classes of numbers $n$. We recall that an integer $n$ is said to be square free if for every prime divisor $q$ of $n$ we have $q^{2} \nmid n$ [1].

Theorem 1.9 $\operatorname{Robins}(n)$ holds for all natural numbers $n>5040$ that are square free [1].

Let $q_{1}=2, q_{2}=3, \ldots, q_{m}$ be the first $m$ consecutive primes, then an integer of the form $\prod_{i=1}^{m} q_{i}^{a_{i}}$ with $a_{1} \geq a_{2} \geq \cdots \geq a_{m} \geq 0$ is called an Hardy-Ramanujan integer [1]. Based on the theorem 1.8, we know this result:

Theorem 1.10 If the Riemann hypothesis is false, then there exist infinitely many natural numbers $n>5040$ which are an Hardy-Ramanujan integer and Robins $(n)$ does not hold [1].

Putting all together yields the proof that there are not infinitely many natural numbers $n>5040$ which are an Hardy-Ramanujan integer and Robins $(n)$ does not hold. Consequently, the Riemann hypothesis is true.

\section{Ancillary lemmas}

The following is a key lemma. It gives an upper bound on $f(n)$ that holds for all natural numbers $n$. The bound is too weak to prove Robins $(n)$ directly, but is critical because it holds for all natural numbers $n$. Further the bound only uses the primes that divide $n$ and not how many times they divide $n$.

Lemma 2.1 Let $n>1$ and let all its prime divisors be $q_{1}<\cdots<q_{m}$. Then,

$$
f(n)<\frac{\pi^{2}}{6} \times \prod_{i=1}^{m} \frac{q_{i}+1}{q_{i}} .
$$

Proof Putting together the theorems 1.7 and 1.4 yields the proof:

$$
f(n)<\prod_{i=1}^{m}\left(\frac{q_{i}}{q_{i}-1}\right)=\prod_{i=1}^{m}\left(\frac{q_{i}+1}{q_{i}} \times \frac{1}{1-\frac{1}{q_{i}^{2}}}\right)<\frac{\pi^{2}}{6} \times \prod_{i=1}^{m} \frac{q_{i}+1}{q_{i}} .
$$


The following is another key lemma.

Lemma 2.2 There exists a natural number $N$ such that $X_{n}<\frac{e^{\gamma} \times 6}{\pi^{2}}+\varepsilon$ for all natural numbers $n>N$ and for a positive real number $\varepsilon<\frac{6}{\pi^{2}}$. Only a finite number of elements of the sequence are greater than $\frac{e^{\gamma} \times 6}{\pi^{2}}+\varepsilon$ (this could be an empty set).

Proof The limit superior of a sequence of real numbers $y_{n}$ is the smallest real number $b$ such that, for any positive real number $\varepsilon$, there exists a natural number $N$ such that $y_{n}<b+\varepsilon$ for all natural numbers $n>N$. Only a finite number of elements of the sequence are greater than $b+\varepsilon$ (this could be an empty set). Therefore, this is a consequence of the theorem 1.2.

\section{A Simple Case}

We can easily prove that $\operatorname{Robins}(n)$ is true for certain kind of numbers:

Lemma 3.1 Robins $(n)$ holds for all natural numbers $n>5040$ when $q \leq 5$, where $q$ is the largest prime divisor of $n$.

Proof Let $n>5040$ and let all its prime divisors be $q_{1}<\cdots<q_{m} \leq 5$, then we need to prove

$$
f(n)<e^{\gamma} \times \log \log n
$$

that is true when

$$
\prod_{i=1}^{m} \frac{q_{i}}{q_{i}-1} \leq e^{\gamma} \times \log \log n
$$

according to the theorem 1.7. For the prime divisors $q_{1}<\cdots<q_{m} \leq 5$,

$$
\prod_{i=1}^{m} \frac{q_{i}}{q_{i}-1} \leq \frac{2 \times 3 \times 5}{1 \times 2 \times 4}=3.75<e^{\gamma} \times \log \log (5040) \approx 3.81
$$

For all natural numbers $n>5040$, we note that

$$
e^{\gamma} \times \log \log (5040)<e^{\gamma} \times \log \log n
$$

and therefore, the proof is complete when $q_{1}<\cdots<q_{m} \leq 5$.

\section{Inequalities on Hardy-Ramanujan integers}

Lemma 4.1 Let $\prod_{i=1}^{m} q_{i}^{a_{i}}$ be the representation of an Hardy-Ramanujan integer $n>$ 5040 as a product of the first $m$ primes $q_{1}<\cdots<q_{m}$ with natural numbers as exponents $a_{1} \geq a_{2} \geq \cdots \geq a_{m} \geq 0$. If Robins $(n)$ does not hold, then $\operatorname{Nicolas}\left(q_{m}\right)$ holds indeed. 
Proof When $\operatorname{Robins}(n)$ does not hold, then

$$
f(n) \geq e^{\gamma} \times \log \log n .
$$

Let's assume that $\operatorname{Nicolas}\left(q_{m}\right)$ does not hold as well. Consequently,

$$
\prod_{q \leq q_{m}} \frac{q}{q-1} \leq e^{\gamma} \times \log \log N_{m} .
$$

According to the theorem 1.7,

$$
\begin{aligned}
e^{\gamma} \times \log \log N_{m} & \geq \prod_{q \leq q_{m}} \frac{q}{q-1} \\
& >f(n) \\
& \geq e^{\gamma} \times \log \log n .
\end{aligned}
$$

However, this implies that $N_{m}>n$ which is a contradiction since $n>5040$ is an Hardy-Ramanujan integer.

\section{When the Nicolas inequality may fail}

Lemma 5.1 If some prime number $q_{n}>2$ complies with

$$
X_{n} \leq \frac{e^{\gamma} \times 6}{\pi^{2}}
$$

then $\operatorname{Nicolas}\left(q_{n}\right)$ does not hold.

Proof If we have the inequality

$$
X_{n} \leq \frac{e^{\gamma} \times 6}{\pi^{2}}
$$

then this is equivalent to

$$
\prod_{q \leq q_{n}} \frac{q+1}{q} \leq \frac{e^{\gamma} \times 6}{\pi^{2}} \times \log \theta\left(q_{n}\right) .
$$

If we multiply the both sides by $\frac{\pi^{2}}{6}$, so

$$
\frac{\pi^{2}}{6} \times \prod_{q \leq q_{n}} \frac{q+1}{q} \leq e^{\gamma} \times \log \theta\left(q_{n}\right) .
$$

We use that theorem 1.4 to show that

$$
\frac{\pi^{2}}{6} \times \prod_{q \leq q_{n}} \frac{q+1}{q}>\left(\prod_{q \leq q_{n}} \frac{q^{2}}{q^{2}-1}\right) \times \prod_{q \leq q_{n}} \frac{q+1}{q} .
$$


Besides,

$$
\left(\prod_{q \leq q_{n}} \frac{q^{2}}{q^{2}-1}\right) \times \prod_{q \leq q_{n}} \frac{q+1}{q}=\prod_{q \leq q_{n}} \frac{q}{q-1}
$$

because of

$$
\frac{q}{q-1}=\frac{q^{2}}{q^{2}-1} \times \frac{q+1}{q} .
$$

Consequently, we obtain that

$$
\prod_{q \leq q_{n}} \frac{q}{q-1} \leq e^{\gamma} \times \log \theta\left(q_{n}\right)
$$

and therefore, Nicolas $\left(q_{n}\right)$ does not hold.

\section{Main Insight}

The next lemma is a main insight.

Lemma 6.1 Let $\frac{\pi^{2}}{6} \times \log \log n^{\prime} \leq \log \log n$ for some natural number $n>5040$ such that $n^{\prime}$ is the square free kernel of the natural number $n$. Then Robins $(n)$ holds.

Proof Let $n^{\prime}$ be the square free kernel of the natural number $n$, that is the product of the distinct primes $q_{1}, \ldots, q_{m}$. By assumption we have that

$$
\frac{\pi^{2}}{6} \times \log \log n^{\prime} \leq \log \log n
$$

For all square free $n^{\prime} \leq 5040$, Robins $\left(n^{\prime}\right)$ holds if and only if $n^{\prime} \notin\{2,3,5,6,10,30\}$ [1]. Robins $(n)$ holds for all natural numbers $n>5040$ when $n^{\prime} \in\{2,3,5,6,10,15,30\}$ due to the lemma 3.1. When $n^{\prime}>5040$, we know that Robins $\left(n^{\prime}\right)$ holds and so

$$
f\left(n^{\prime}\right)<e^{\gamma} \times \log \log n^{\prime}
$$

because of the theorem 1.9. By the previous lemma 2.1:

$$
f(n)<\frac{\pi^{2}}{6} \times \prod_{i=1}^{m} \frac{q_{i}+1}{q_{i}} .
$$

Suppose by way of contradiction that $\operatorname{Robins}(n)$ fails. Then

$$
f(n) \geq e^{\gamma} \times \log \log n .
$$

We claim that

$$
\frac{\pi^{2}}{6} \times \prod_{i=1}^{m} \frac{q_{i}+1}{q_{i}}>e^{\gamma} \times \log \log n .
$$

Since otherwise we would have a contradiction. This shows that

$$
\frac{\pi^{2}}{6} \times \prod_{i=1}^{m} \frac{q_{i}+1}{q_{i}}>\frac{\pi^{2}}{6} \times e^{\gamma} \times \log \log n^{\prime} .
$$


Thus

$$
\prod_{i=1}^{m} \frac{q_{i}+1}{q_{i}}>e^{\gamma} \times \log \log n^{\prime}
$$

and

$$
\prod_{i=1}^{m} \frac{q_{i}+1}{q_{i}}>f\left(n^{\prime}\right)
$$

This is a contradiction since $f\left(n^{\prime}\right)$ is equal to

$$
\frac{\left(q_{1}+1\right) \times \cdots \times\left(q_{m}+1\right)}{q_{1} \times \cdots \times q_{m}}
$$

according to the formula $f(x)$ for the square free numbers [1].

\section{Proof of the Riemann Hypothesis}

Theorem 7.1 The Riemann hypothesis is true.

Proof Let $\prod_{i=1}^{m} q_{i}^{a_{i}}$ be the representation of a sufficiently large Hardy-Ramanujan integer $n>5040$ as a product of the first $m$ primes $q_{1}<\cdots<q_{m}$ with natural numbers as exponents $a_{1} \geq a_{2} \geq \cdots \geq a_{m} \geq 0$. We claim that for every sufficiently large Hardy-Ramanujan integer $n>5040$, then Robins $(n)$ could always hold. Suppose that Robins $(n)$ does not hold and so, the Riemann hypothesis would be false. Hence,

$$
f(n) \geq e^{\gamma} \times \log \log n .
$$

We use that theorem 1.6,

$$
\left(\prod_{i=1}^{m} \frac{q_{i}}{q_{i}-1}\right) \times \prod_{i=1}^{m}\left(1-\frac{1}{q_{i}^{a_{i}+1}}\right) \geq e^{\gamma} \times \log \log n
$$

which is equivalent to

$$
\left(\prod_{i=1}^{m} \frac{q_{i}^{2}}{q_{i}^{2}-1}\right) \times\left(\prod_{i=1}^{m} \frac{q_{i}+1}{q_{i}}\right) \times \prod_{i=1}^{m}\left(1-\frac{1}{q_{i}^{a_{i}+1}}\right) \geq e^{\gamma} \times \log \log n .
$$

If we divide the both sides by $\log \log N_{m}$, then we obtain

$$
\left(\prod_{i=1}^{m} \frac{q_{i}^{2}}{q_{i}^{2}-1}\right) \times X_{m} \times \prod_{i=1}^{m}\left(1-\frac{1}{q_{i}^{a_{i}+1}}\right) \geq e^{\gamma} \times \frac{\log \log n}{\log \log N_{m}}
$$

because of $\log \log N_{m}=\log \theta\left(q_{m}\right)$, where $N_{m}$ is the primorial number of order $m$. We know that $X_{m} \leq \frac{e^{\gamma} \times 6}{\pi^{2}}$ is false according to the lemmas 4.1 and 5.1. From the lemma 2.2, we know that there exists a natural number $N$ such that $X_{m}<\frac{e^{\gamma} \times 6}{\pi^{2}}+\varepsilon$ for all natural numbers $m>N$ and for a positive real number $\varepsilon<\frac{6}{\pi^{2}}$. Moreover, only a finite number of elements of the sequence are greater than $\frac{e^{\gamma} \times 6}{\pi^{2}}+\varepsilon$ (this could be an empty set). Under our assumption, there exist infinitely many Hardy-Ramanujan 
integers $n>5040$ such that Robins $(n)$ does not hold and $X_{m}<\frac{e^{\gamma} \times 6}{\pi^{2}}+\varepsilon$. In addition, $q_{m}$ cannot have an upper bound under our assumption. In general, if $q_{m}$ would have an upper bound, then our assumption fails as a consequence of the lemma 6.1. In this way, we obtain that

$$
\left(\prod_{i=1}^{m} \frac{q_{i}^{2}}{q_{i}^{2}-1}\right) \times\left(\frac{e^{\gamma} \times 6}{\pi^{2}}+\varepsilon\right) \times \prod_{i=1}^{m}\left(1-\frac{1}{q_{i}^{a_{i}+1}}\right) \geq e^{\gamma} \times \frac{\log \log n}{\log \log N_{m}}
$$

which is the same as

$$
\left(\prod_{i=1}^{m} \frac{q_{i}^{2}}{q_{i}^{2}-1}\right) \times \frac{6}{\pi^{2}} \times\left(e^{\gamma}+c\right) \times \prod_{i=1}^{m}\left(1-\frac{1}{q_{i}^{a_{i}+1}}\right) \geq e^{\gamma} \times \frac{\log \log n}{\log \log N_{m}}
$$

for a sufficiently small positive value of $c=\varepsilon \times \frac{\pi^{2}}{6}$. That would be equivalent to

$$
\left(\prod_{q>q_{m}} \frac{q^{2}-1}{q^{2}}\right) \times\left(e^{\gamma}+c\right) \times \prod_{i=1}^{m}\left(1-\frac{1}{q_{i}^{a_{i}+1}}\right) \geq e^{\gamma} \times \frac{\log \log n}{\log \log N_{m}} .
$$

Since $n$ is an Hardy-Ramanujan integer, then

$$
\left(\prod_{q>q_{m}} \frac{q^{2}-1}{q^{2}}\right) \times \prod_{i=1}^{m}\left(1-\frac{1}{q_{i}^{a_{i}+1}}\right)<\prod_{q}\left(1-\frac{1}{q^{a_{1}+1}}\right)=\frac{1}{\zeta\left(a_{1}+1\right)}
$$

because of the theorem 1.5 , where $a_{1}$ is the highest exponent such that $2^{a_{1}} \mid n$. Therefore,

$$
\frac{\left(e^{\gamma}+c\right)}{\zeta\left(a_{1}+1\right)}>e^{\gamma} \times \frac{\log \log n}{\log \log N_{m}}
$$

for a sufficiently small positive value of $0<c<1$. However, this could be false for a sufficiently small positive value of $\varepsilon<\frac{6}{\pi^{2}}$ that we could choose, where $c=\varepsilon \times \frac{\pi^{2}}{6}$ would be a very small positive value as well. In addition, we know that $\frac{\log \log n}{\log \log N_{m}}>1$ due to the theorem 1.9. Furthermore, from the paper [3], we know that Robins $(n)$ holds for all natural numbers $n>5040$ when

$$
a_{1}>\left\lceil\frac{1}{\log 2} \times\left(\left(\log \left(2^{-a_{1}+19} \times n\right)\right)^{\frac{1048576}{1048575}}-\log \left(2^{-a_{1}} \times n\right)\right)\right\rceil .
$$

In conclusion, for every sufficiently large Hardy-Ramanujan integer $n>5040$, then Robins $(n)$ could always hold. By contraposition, the Riemann hypothesis is true, because of the theorems 1.8 and 1.10 .

\section{Acknowledgments}

The author wishes to thank Richard J. Lipton and Craig Helfgott for helpful comments and my mother, maternal brother and my friend Sonia for their support. 


\section{References}

1. Choie, Y., Lichiardopol, N., Moree, P., Solé, P.: On Robin's criterion for the Riemann hypothesis. Journal de Théorie des Nombres de Bordeaux 19(2), 357-372 (2007). DOI 10.5802/jtnb.591

2. Edwards, H.M.: Riemann's Zeta Function. Dover Publications (2001)

3. Hertlein, A.: Robin's inequality for new families of integers (2016). ArXiv math.NT 1612.05186v1 at https://arxiv.org/pdf/1612.05186v1.pdf

4. Nicolas, J.L.: Petites valeurs de la fonction d'Euler. Journal of number theory 17(3), 375-388 (1983). DOI 10.1016/0022-314X(83)90055-0

5. Robin, G.: Grandes valeurs de la fonction somme des diviseurs et hypothèse de Riemann. J. Math. pures appl 63(2), 187-213 (1984) 